\title{
Supporting information for Time evolution of the behaviour of Brazilian legislative Representatives using a complex network approach.
}

\section{Data mining and manipulation.}

The data mining consisted of processing individual files containing the results of the rollcall votes of Deputies for each session over 16 years. After identifying each elected Deputy in all sessions per year, a file containing each Deputy name and an associated array of her/his votes, was obtained for each year. Special care was taken to account for partial and total absence frequency of a Deputy in each year. The partial absence zones were filled using information about substitutes for each Deputy. If the information about the substitute of Deputy was scarce, we assume that substitute was a member of the same political party and then, if the absence zones and vote zones coincide, the substitute vote is computed. Deputies with total absences throughout the year without certified substitute were eliminated. The quantity of Deputies and bills after the data processing for each year is shown in Table S1.

\begin{tabular}{|c|c|c|}
\hline Year & Deputies & Bills \\
\hline \hline 2003 & 415 & 151 \\
2004 & 399 & 106 \\
2005 & 375 & 79 \\
2006 & 368 & 96 \\
2007 & 499 & 219 \\
2008 & 492 & 150 \\
2009 & 472 & 165 \\
2010 & 477 & 89 \\
\hline
\end{tabular}

\begin{tabular}{|c|c|c|}
\hline Year & Deputies & Bills \\
\hline \hline 2011 & 481 & 101 \\
2012 & 466 & 82 \\
2013 & 462 & 161 \\
2014 & 455 & 88 \\
2015 & 481 & 300 \\
2016 & 400 & 222 \\
2017 & 397 & 236 \\
2018 & 391 & 149 \\
\hline
\end{tabular}

Table S1: Quantity of Deputies and bills after the transfer of absence zones at the Brazilian Chamber of Deputies from 2003 to 2018. Absences were completed with the certified substitute.

\section{The Minimal Spanning Tree (MST).}

We use a weighted graph $G(V, E, W, f)$ for designing all networks. We employ the Prim's algorithm to obtain the MST. The Prim algorithm $j^{\text {th }}$ step consists of finding the closest pair of vertices to set of $(j-1)^{t h}$ MST sub network $N_{j-1}$ for each only one vertex, but not the other, belongs to $N_{j-1}$. This way the vertex that did not belong to $N_{j-1}$ is 
now incorporated into the $N_{j}$ MST subnetwork. It does not matter that another much closer pair of vertices exists, but none of the vertices are yet connected. As the algorithm interacts in a complete network, eventually, all pairs of vertices will be connected. This shows that MST connections can be the best way to connect the closest neighbours and display the clusters in the correlation, or distance, matrices. Our procedure, therefore, was to use the correlation matrix to obtain the MST and then reorder rows and columns of the correlation matrix with the MST-prim order. All MST networks were designed using the software package Pajek and we rearrange vertices manually to avoid much line crossing. Fig S1 shows the optimize Deputies' network from 2003 to 2018. 


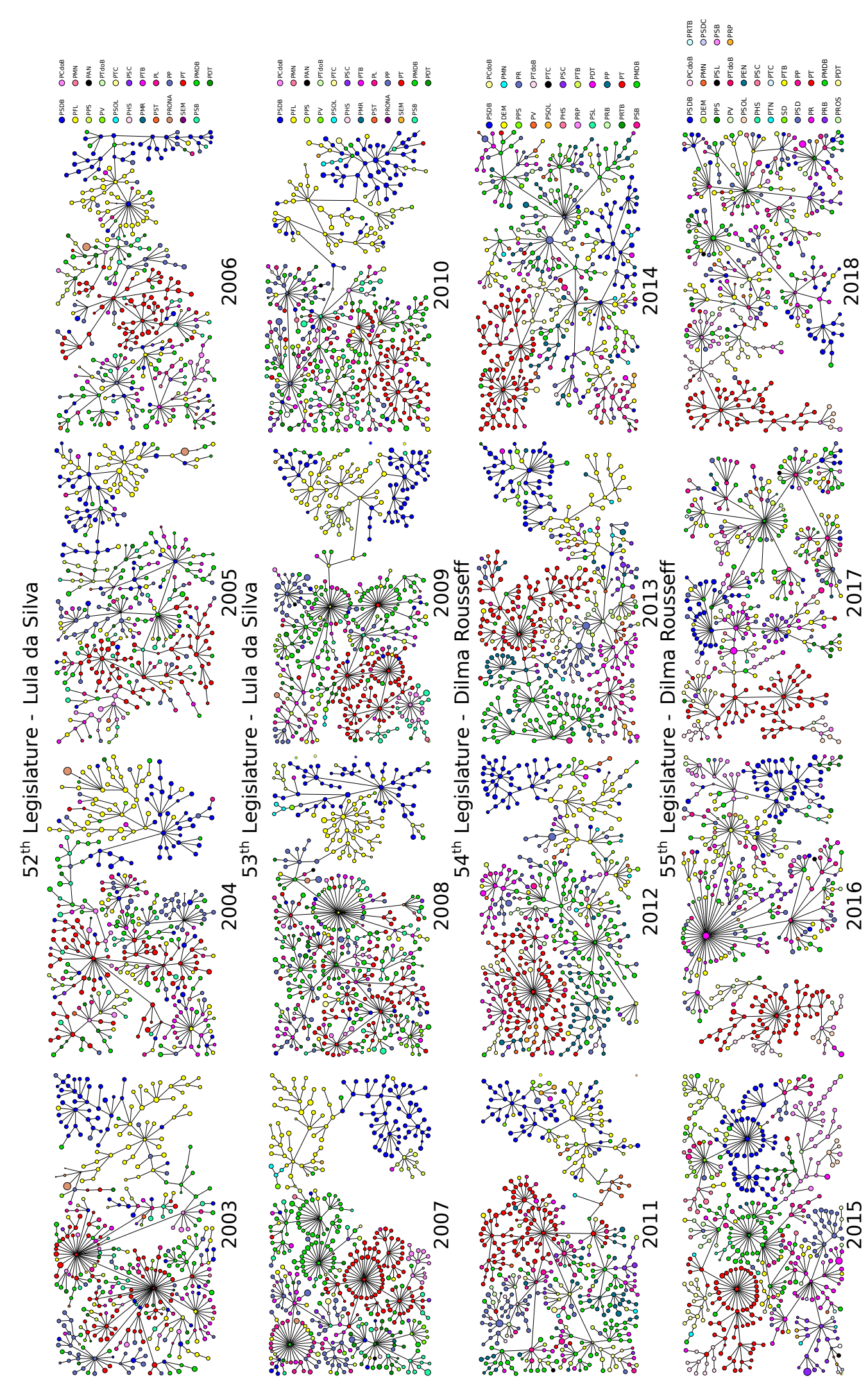

Fig S1: Representatives' networks from 2003 to 2018. Each vertex of the MST corresponds to a Deputy. Two attributes was assigned to the vertex set: the colour cluster is associated with the political party and the size of each vertex is a measure of the quantity of votes by which each Deputy was elected. 


\section{Correlation distribution fitting.}

The fitting was made over the cumulative distribution because this distribution is independent of the choice of the bin range. The procedure was carried out by minimizing the sum of residual squared between the fitting function and the experimental data. Table $\mathrm{S} 2$ shows the fitted parameters of the cumulative distribution for each year.

\begin{tabular}{|c|c|c|c|c|c|c|}
\hline Year & $b$ & $\mu_{1}$ & $\mu_{2}$ & $\sigma_{1}$ & $\sigma_{2}$ & $R^{2}$ \\
\hline \hline 2003 & 0.4402 & 0.0816 & 0.6177 & 0.1055 & 0.0943 & 0.9984 \\
2004 & 0.5491 & 0.2427 & 0.5635 & 0.0954 & 0.0830 & 0.9999 \\
2005 & 0.4275 & 0.5246 & 0.2109 & 0.0810 & 0.0993 & 0.9999 \\
2006 & 0.5131 & 0.2683 & 0.5374 & 0.0831 & 0.0808 & 0.9998 \\
2007 & 0.4003 & -0.0887 & 0.6302 & 0.119 & 0.1386 & 0.9992 \\
2008 & 0.3951 & 0.0353 & 0.5911 & 0.1215 & 0.1463 & 0.9998 \\
2009 & 0.4259 & 0.1338 & 0.4845 & 0.1225 & 0.1286 & 1 \\
2010 & 0.5655 & 0.2596 & 0.4936 & 0.163 & 0.1283 & 0.9999 \\
2011 & 0.3757 & 0.0029 & 0.6138 & 0.2387 & 0.1482 & 0.999 \\
2012 & 0.3751 & 0.2086 & 0.4303 & 0.1574 & 0.1525 & 1 \\
2013 & 0.8386 & 0.3628 & 0.1935 & 0.1407 & 0.1171 & 1 \\
2014 & 0.5043 & 0.4430 & 0.2844 & 0.1332 & 0.1368 & 1 \\
2015 & 0.9125 & 0.1921 & 0.4152 & 0.2346 & 0.0997 & 1 \\
2016 & 0.5965 & 0.5418 & 0.6582 & 0.0744 & 0.0886 & 0.9998 \\
2017 & 0.4164 & 0.1098 & 0.4801 & 0.0795 & 0.0892 & 0.9998 \\
2018 & 0.4975 & 0.5371 & 0.1407 & 0.0863 & 0.0893 & 0.9997 \\
\hline
\end{tabular}

Table S2: Fitted parameters for the correlation distribution for $52^{\text {th }}, 53^{\text {th }}, 54^{\text {th }}$ and $55^{\text {th }}$ Legislature of the Brazilian Chamber of Deputies.

\section{$4 \quad$ Null Model}

The null model was created by shuffling the roll-call vote matrices for all years. The shuffling was performed by selecting a source node, creating a list with the indexes of all connected nodes (original targets, in this case all other nodes in the network) in a random order, and replacing each edge connecting with the original targets with the ones from the random list. This process is repeated for all nodes. Fig S2 shows the MST sub-network and MST-ordered correlation matrices after the shuffling process for the $53^{\text {th }}$ Legislature. 


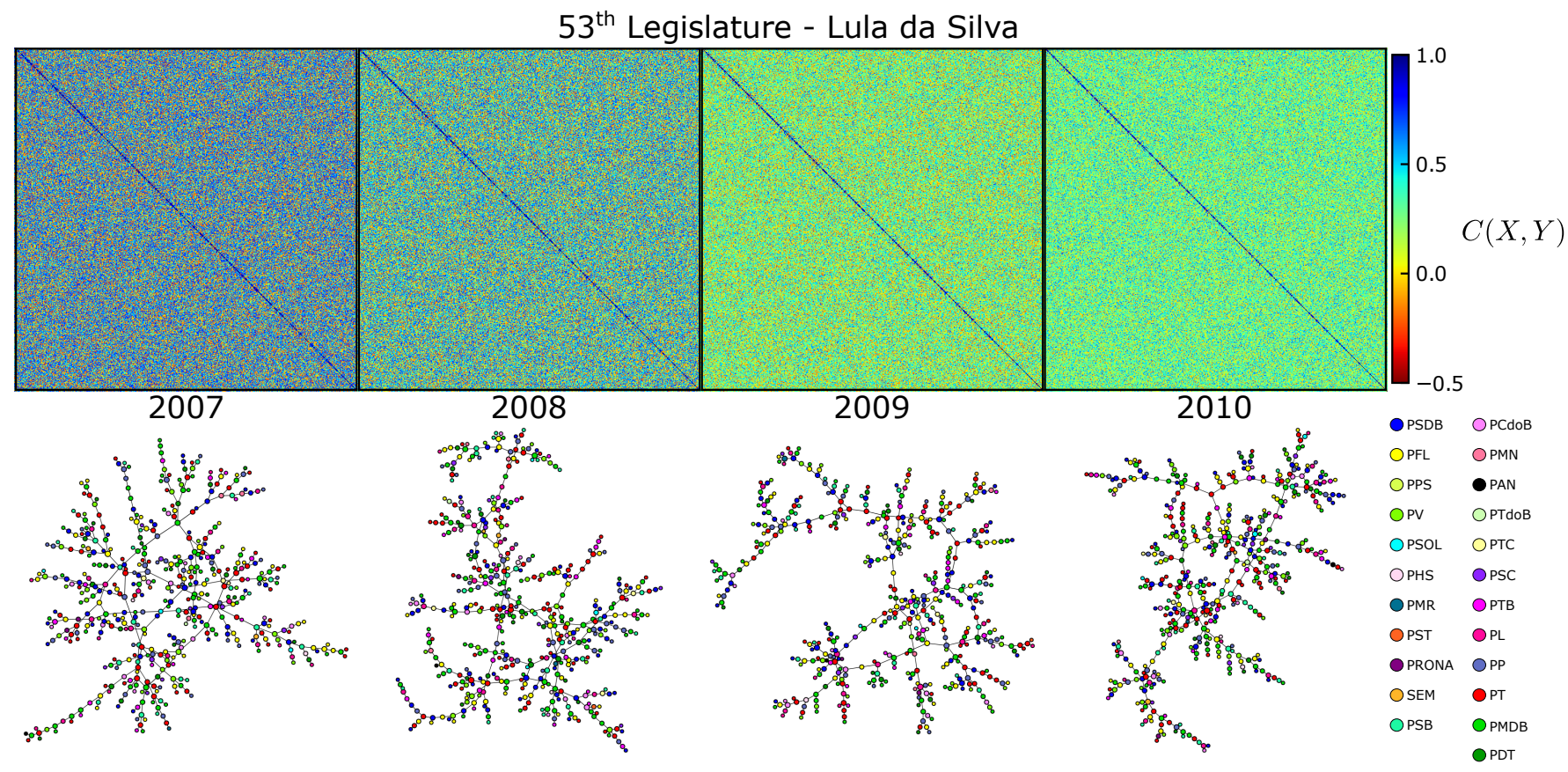

Fig S2: MST sub-network and MST-reordered correlation matrices for $53^{\text {th }}$ Legislature for the Null Model of the Brazilian Chamber of Deputies. 